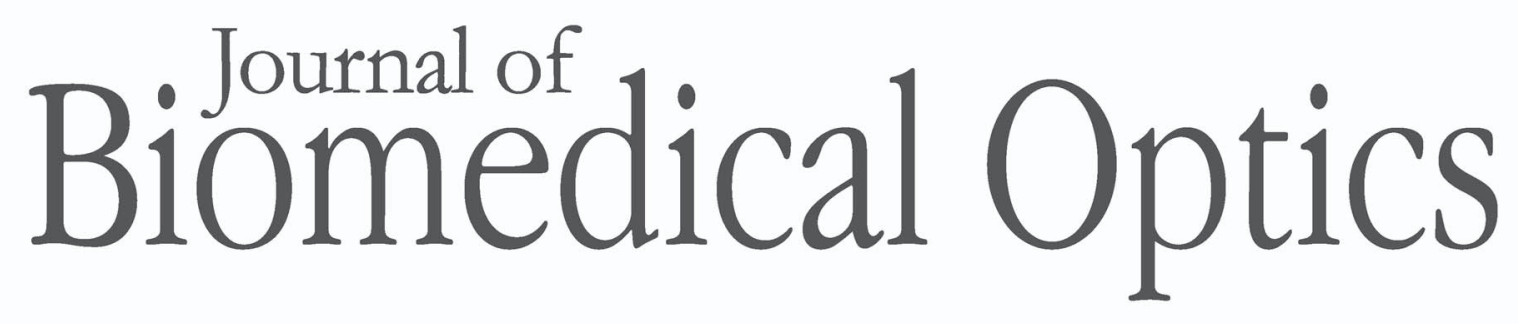

\title{
Spontaneous ultraweak photon emission imaging of oxidative metabolic processes in human skin: effect of molecular oxygen and antioxidant defense system
}

Anshu Rastogi

Pavel Pospíšil 


\title{
Spontaneous ultraweak photon emission imaging of oxidative metabolic processes in human skin: effect of molecular oxygen and antioxidant defense system
}

\author{
Anshu Rastogi and Pavel Pospišil \\ Palacký University, Department of Biophysics, Centre of the Region Haná for Biotechnological and Agricultural \\ Research, Faculty of Science, Šlechtitelů 11, 78371 Olomouc, Czech Republic
}

\begin{abstract}
All living organisms emit spontaneous ultraweak photon emission as a result of cellular metabolic processes. In this study, the involvement of reactive oxygen species (ROS) formed as the byproduct of oxidative metabolic processes in spontaneous ultraweak photon emission was studied in human hand skin. The effect of molecular oxygen and ROS scavengers on spontaneous ultraweak photon emission from human skin was monitored using a highly sensitive photomultiplier tube and charged coupled device camera. When spontaneous ultraweak photon emission was measured under anaerobic conditions, the photon emission was decreased, whereas under hyperaerobic condition the enhancement in photon emission was observed. Spontaneous ultraweak photon emission measured after topical application of glutathione, $\alpha$-tocopherol, ascorbate, and coenzyme Q10 was observed to be decreased. These results reveal that ROS formed during the cellular metabolic processes in the epidermal cells play a significant role in the spontaneous ultraweak photon emission. It is proposed that spontaneous ultraweak photon emission can be used as a noninvasive tool for the temporal and spatial monitoring of the oxidative metabolic processes and intrinsic antioxidant system in human skin. $\odot 2011$ Society of Photo-Optical Instrumentation Engineers (SPIE). [DOI: 10.1117/1.3616135]
\end{abstract}

Keywords: antioxidant; molecular oxygen; reactive oxygen species; ultraweak photon emission.

Paper 11193R received Apr. 15, 2011; revised manuscript received Jul. 1, 2011; accepted for publication Jul. 7, 2011; published online Sep. 1, 2011.

\section{Introduction}

Metabolic processes are the fundamental chemical reactions occurring inside the living cells. It is known that metabolic processes (e.g., cellular respiration, oxidative burst) result in the generation of reactive oxygen species (ROS) known to play an important role in the defense against infection, cell signaling, apoptosis, and aging. ${ }^{1,2}$ However, due to the high positive redox potential, ROS have a capability to oxidize various cellular components such as lipids, proteins, and nucleic acids. ${ }^{2-5}$ To prevent the oxidative damage of cellular components, the cells developed their own defense system for the protection against ROS. Under certain circumstances, when the formation of ROS exceeds the capacity of an antioxidant system, the dangerous ROS are insufficiently scavenged. The imbalance between formation and scavenging of ROS brings about the damage of cellular membrane known to lead to the death of the cell.

In human skin, superoxide anion radical $\left(\mathrm{O}_{2}{ }^{-}\right)$is formed by one-electron reduction of molecular oxygen catalyzed by various mitochondrial and cytoplasmic enzymes. In the mitochondrial membrane, the leakage of electron from complexes I and III within the electron transport chain leads to the formation of $\mathrm{O}_{2} \cdot{ }^{-6} \cdot{ }^{6,7}$ More recent evidence reveals that complex II in the mitochondrial membrane also contributes to the overall $\mathrm{O}_{2} \cdot-$ production in human skin cells. ${ }^{8}$ Under physiological conditions, up to $5 \%$ of molecular oxygen consumed by mi-

Address all correspondence to: Pavel Pospíšil, Palacký University, Centre of the Region Haná for Biotechnological and Agricultural Research, Department of Biophysics, Faculty of Science, Šlechtitelů 11, 78371 Olomouc, Czech Republic; Tel.: + 420 585634174; Fax: + 420 585225737; E-mail: pospip@prfnw.upol.cz. tochondria is reduced to $\mathrm{O}_{2} \cdot{ }^{-} \cdot{ }^{9}$ Apart from the mitochondria, $\mathrm{O}_{2} \cdot{ }^{-}$is formed in the cytoplasm of skin keratinocyte cells by a reduction of molecular oxygen catalyzed by heme-flavoprotein NADPH oxidase (NOX). ${ }^{10}$

Various types of nonenzymatic and enzymatic antioxidant systems are engaged to eliminate the cellular oxidative damage. ${ }^{11}$ Nonenzymatic antioxidant systems in human skin include ascorbate, $\alpha$-tocopherol, carotenoids, gluthatione, and coenzyme Q10, whereas enzymatic scavenging is maintained by enzymes such as the superoxide dismutase (SOD) family of enzymes (copper-zinc SOD, manganese SOD) and the peroxidase family of enzymes (glutathione peroxidases and catalase). ${ }^{1-13}$ When the formation of ROS overwhelms the skin antioxidant capacity, the subsequent oxidation of lipids, proteins, and nucleic acids leads to lipid peroxidation, protein degradation, and nucleic acid damage, ${ }^{14}$ which cause the aging of the skin.

The oxidation of lipids, proteins, and nucleic acids by ROS is accompanied by the formation of electronically excited species such as triplet carbonyls ${ }^{3}(\mathrm{C}=\mathrm{O}){ }^{*}$ and singlet oxygen $\left({ }^{1} \mathrm{O}_{2}\right) \cdot{ }^{15,16}$ The light emission of the electronically excited species in the spectral range from 200 to $800 \mathrm{~nm}$ is known as weak chemiluminescence often called ultraweak photon emission. ${ }^{17-21}$ Typically, the intensity of photon emission is less than approximately $10^{-15} \mathrm{~W} / \mathrm{cm}^{2}$ on the surface of human skin. It is known that different wavelengths of light have different penetration depths in human skin. ${ }^{22}$ Due to its ultraweak nature and emission in the visible range of the spectrum, it is possible to detect the

1083-3668/2011/16(9)/096005/7/\$25.00 @ 2011 SPIE 
ultraweak photon emission only from the $3 \mathrm{~mm}$ layer below the body surface. ${ }^{23}$ It indicates that the oxidative metabolic processes in the skin play a significant role in the overall photon emission from the human body.

Apart from ultraweak photon emission, other noninvasive techniques such as attenuated total reflection-Flourier transform infrared spectroscopy (ATR-FTIR), resonance Raman spectroscopy, and confocal Raman microscopy can be used for the measurement of oxidative stress in human skin. For the noninvasive detection of lipid composition from stratum corneum, ATR-FTIR can be used. ${ }^{24}$ On the other hand, resonance Raman spectroscopy can noninvasively detect the antioxidant level of human skin, ${ }^{25,26}$ whereas confocal Raman microscopy can be used for noninvasive depth-resolved assessment of specific antioxidants. ${ }^{27,28}$ The ultraweak photon emission or weak chemiluminescence is the most frequent noninvasive technique used for the measurement of oxidative stress because of its direct correlation with the oxidative metabolic processes. ${ }^{29-35}$

The effect of scavengers on ultraweak photon emission was studied in human skin exposed to various types of physical (ultraviolet and ultrasonic radiation) and chemical (hydrogen peroxide, ozone, cigarette smoke) stress factors. ${ }^{30-33}$ It has been demonstrated that topical application of ascorbic acid prior to exposure of human skin to ultraviolet radiation (UV) significantly reduced UV-induced ultraweak photon emission. ${ }^{33}$ More recently, a topical application of antioxidant system (tocopheryl acetate, ferulic acid, and rutin) on human skin has been shown to significantly decrease UV-induced ultraweak photon emission. ${ }^{29}$ Hagens et al. (2008) demonstrated that exposure of human skin to ozone and cigarette smoke caused an enhancement in ultraweak photon emission, whereas topical application of antioxidant (ascorbate and $\alpha$-glucosylrutin) to human skin reduced ultraweak photon emission. ${ }^{31}$

Whereas the application of ultraweak photon emission for the detection of the oxidative metabolic processes in human skin exposed to physical and chemical stress factors is widely used, the application of spontaneous ultraweak photon emission for the detection of oxidative processes in the skin the related to the normal metabolic processes, i.e., without the use of any exogenous stress factor has received less attention. As far as we know, no experimental evidence has been provided on the correlation of spontaneous ultraweak photon emission and oxidative metabolic processes in human skin. The lack of experimental data on the involvement of ROS produced during the normal metabolic processes in spontaneous ultraweak photon emission was due to the insufficient abundance sensitivity of the detection systems in the past. However, the recent development of a highly sensitive device like the photomultiplier tube (PMT) and charged coupled device (CCD) camera has made it possible to detect the spontaneous ultraweak photon emission from human skin. ${ }^{30,34,35}$ Our study, to the best of our knowledge for the first time, shows spontaneous ultraweak photon emission as the tool for the measurement of oxidative and aging processes in human skin.

\section{Material and Method}

\subsection{Human}

Measurements were done on a healthy man of age 25 to 27 years and each measurement was repeated at least three times. The dorsal side of the right hand was used for all measurements.

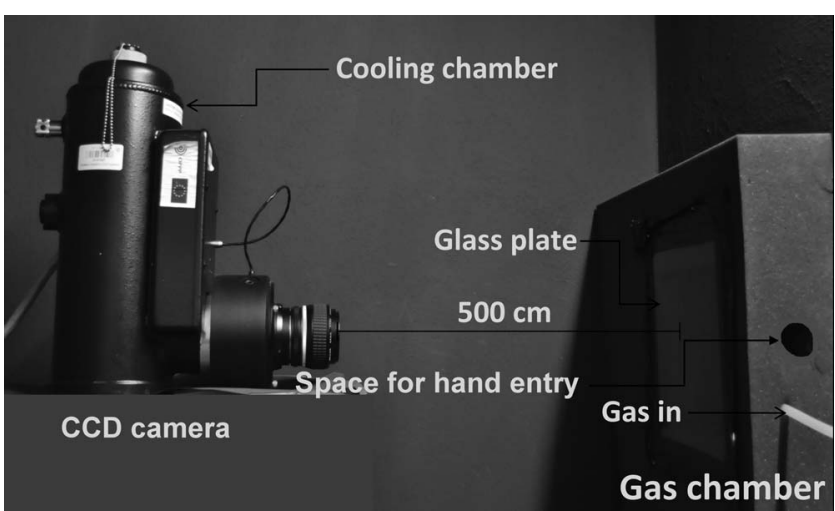

Fig. 1 Experimental setup for the detection of two-dimensional spontaneous ultraweak photon emission under aerobic, anaerobic, and hyperaerobic conditions. The gas chamber is distanced $500 \mathrm{~cm}$ from the CCD window.

The person was adapted to the dark for $20 \mathrm{~min}$ prior to the measurement. The measurement was performed between 11 and $15 \mathrm{~h}$ of the day during the same season to avoid any effect of diurnal and seasonal change. ${ }^{36}$

\subsection{Aerobic, Hyperaerobic, and Anaerobic Conditions}

A special gas chamber designed for the detection of spontaneous ultraweak photon emission from the dorsal side of the hand was used to study the effect of anaerobic and hyperaerobic conditions on ultraweak photon emission. The front side of the gas chamber was made of a glass plate, which allowed the light to penetrate in the visible range of the spectra. The gas chamber was hermetically sealed from the surrounding atmosphere. Prior to the detection of photon emission, a dark-adapted hand was placed inside the chamber faced to the PMT or CCD window at a distance of 10 and $50 \mathrm{~cm}$, respectively (Fig. 1). Nitrogen and oxygen gas was continuously supplied to create the anaerobic and hyperaerobic condition, respectively, whereas the aerobic condition was maintained at the atmospheric air.

\subsection{Topical Application of Scavengers}

Glutathione, ascorbate, $\alpha$-tocopherol, and coenzyme Q10 were used in $5 \mathrm{mM}$ concentration for the study. Scavengers were applied using a micropipette and gloves covering the left hand. For a one-dimensional measurement, a fixed volume of 300 $\mu \mathrm{L}$ of scavengers was used for each measurement and equally distributed to the fixed area of the right hand with the help of the left hand by the subject himself ( 5 min prior to the measurement) in the dark; whereas for a two-dimensional measurement, 1000 $\mu \mathrm{L}$ of scavengers were applied on the dorsal side of the hand. In the control sample, the same volume of water or ethanol was applied. The person was adapted to the dark for 20 min including a 5 min time interval after the application of scavengers.

\subsection{One-Dimensional Ultraweak Photon Emission}

A photon counting unit C9744 (Hamamatsu Photonics K.K., Iwata City, Japan) and photomultiplier tube R7518P (Hamamatsu Photonics K.K., Iwata City, Japan) were used to 
detect the spontaneous ultraweak photon emission from human hand skin. The sensitivity of the instrument was within the range of 185 to $730 \mathrm{~nm}$. To reduce thermal electrons, PMT was cooled to $-30^{\circ} \mathrm{C}$ using a thermoelectric cooler C9143 (Hamamatsu Photonics K.K., Iwata City, Japan). A data equation unit and a computer were placed in an operational dark room. The screen and light emitting diodes were shielded by a black curtain. Inside the operational dark room, an experimental dark room $(3 \times 2 \times 2 \mathrm{~m})$ was built to avoid any effect of the lost photons on PMT. The whole interior in the experimental dark room was painted black and the door was protected from incoming light by a black curtain.

\subsection{Two-Dimensional Ultraweak Photon Emission}

For two-dimensional imaging of spontaneous ultraweak photon emission, a highly sensitive CCD camera VersArray 1300B (Princeton instruments, Trenton, New Jersey) was used. The CCD camera was equipped with a $50-\mathrm{mm}$ focal distance lens with an f-number of 1.2 (F mount Nikkor 50-mm, f:1.2, Nikon, Tokyo, Japan) to enhance the light collecting efficiency. The spectral sensitivity of the CCD camera was within the range of 200 to $1000 \mathrm{~nm}$. Due to the lenses used, the spectral sensitivity of CCD camera was restricted to the visible range of the spectra. To reduce thermal electrons, the CCD camera was cooled to $-110{ }^{\circ} \mathrm{C}$ using liquid nitrogen. The camera was placed in the experimental dark room and controlled by the computer in the operational dark room. The data correction was made by subtracting the background noise before every measurement. The measurement was done in the image format of $1340 \times 1300$ pixels. To improve the image quality the binning mode with the binning factor 4 was applied, which reduces the number of pixels by 4 . CCD camera parameters were as follows: scan rate, $100 \mathrm{kHz}$; gain, 3; accumulation time, $30 \mathrm{~min}$.

\section{Results}

\subsection{One-Dimensional Spontaneous Ultraweak Photon Emission from the Human Hand}

One-dimensional spontaneous ultraweak photon emission from the human hand was measured using a highly sensitive PMT. Figure 2(a) shows that the dark count of PMT was adjusted to 2 counts $\mathrm{s}^{-1}$. When the dorsal side of the hand was placed below the PMT window, the count rate of 4.5 counts s ${ }^{-1}$ was observed [Fig. 2(b)]. After subtraction of the dark count, spontaneous ultraweak photon emission from the dorsal side of the hand was determined as 2.5 counts $\mathrm{s}^{-1}$. When the exogenous environmental conditions in the experimental dark room were unchanged, a spontaneous ultraweak photon emission persisted on the time scale of several hours. These observations reveal that spontaneous ultraweak photon emission connected to the oxidative metabolic processes is an intrinsic property of the epidermal cells in human skin.

\subsection{Effect of Molecular Oxygen on One-Dimensional Spontaneous Ultraweak Photon Emission}

To study the involvement of molecular oxygen in spontaneous ultraweak photon emission from human skin, the photon emission was measured under aerobic, anaerobic, and hyperaerobic
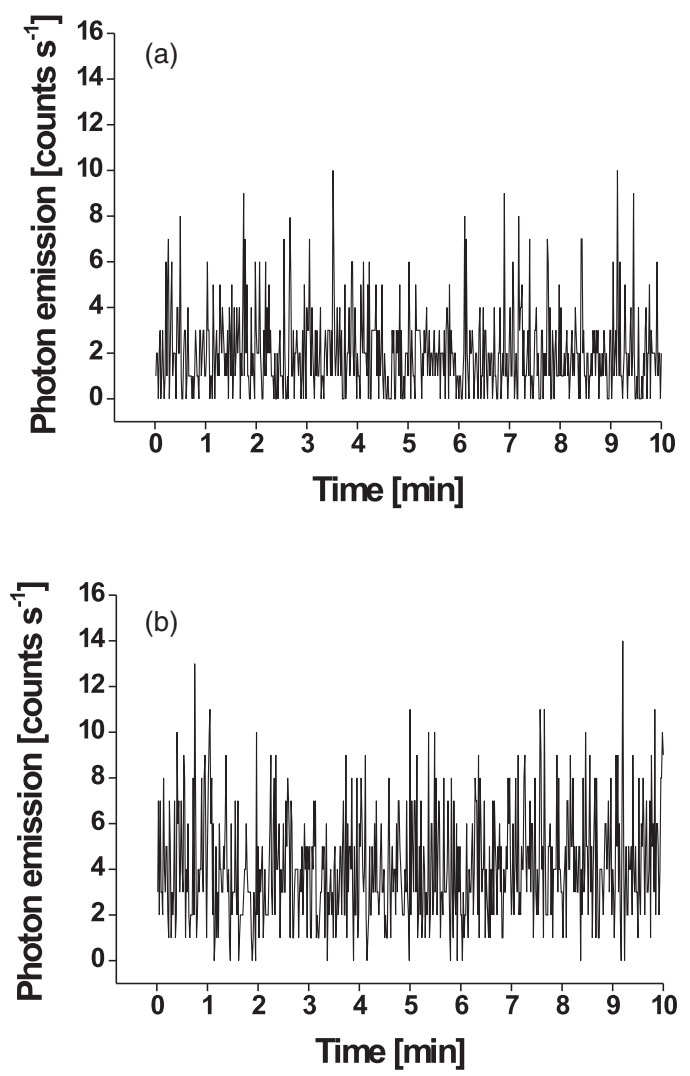

Fig. 2 (a) Dark count rate of the photomultiplier tube and (b) onedimensional spontaneous ultraweak photon emission from the dorsal side of the hand. Before the measurement the subject was adapted to complete darkness for $20 \mathrm{~min}$.

conditions. To control gas environment around the human hand, the human hand was placed in a gas chamber. Under aerobic conditions, the spontaneous ultraweak photon emission from the dorsal side of the hand was 2 counts $\mathrm{s}^{-1}$ (Fig. 3). The slight decrease in the spontaneous ultraweak photon emission observed in the gas chamber, compared to that observed without the gas chamber [Fig. 2(b)], is due to the photon absorption by the glass plate in the glass chamber. When the air in the gas chamber was replaced with nitrogen gas, spontaneous ultraweak photon emission was suppressed to 1.25 counts $\mathrm{s}^{-1}$ (by $40 \%$ compared to aerobic conditions). The supply of molecular oxygen in the gas chamber results in the enhancement in spontaneous ultraweak photon emission to 3 counts s $\mathrm{s}^{-1}$ (by $50 \%$ compared to aerobic conditions). These results reveal that the molecular oxygen is involved in spontaneous ultraweak photon emission from the epidermal cells in human skin.

\subsection{Effect of Molecular Oxygen on Two-Dimensional Spontaneous Ultraweak Photon Emission}

To monitor spatial distribution of photon emission from the human hand, two-dimensional spontaneous ultraweak photon emission was measured using CCD camera. Figure 4 shows the photograph of the dorsal side of the hand and its corresponding photon emission images measured under aerobic, anaerobic, and hyperaerobic conditions. To quantify the differences in sponta- 


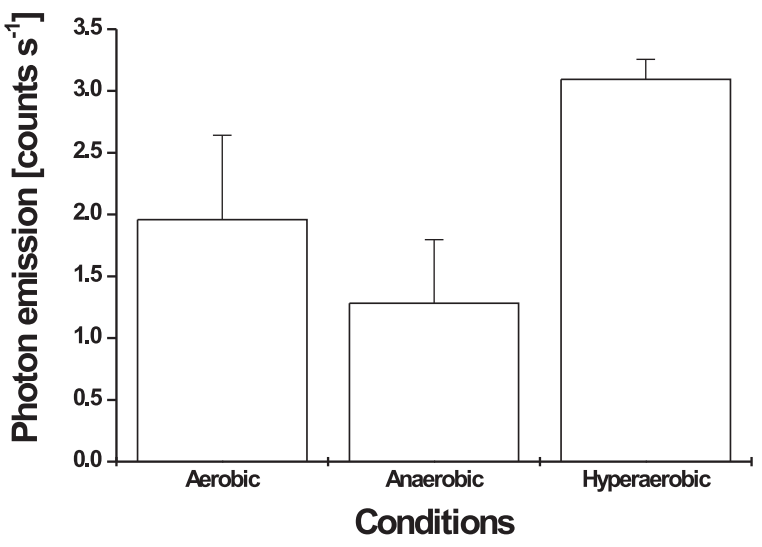

Fig. 3 Effect of molecular oxygen on one-dimensional spontaneous ultraweak photon emission from the dorsal side of the hand. Aerobic condition were established by the supply of the atmospheric air into the gas chamber. Anaerobic conditions were maintained by establishing a continuous flow of nitrogen gas into the gas chamber, whereas molecular oxygen was continuously supplied into the gas chamber to create the hyperaerobic conditions. Spontaneous ultraweak photon emission from human hand was measured for $15 \mathrm{~min}$. Each bar represents the mean value of at least three independent measurements \pm SD made at the same diurnal time of three different days.

neous ultraweak photon emission from the human hand under various concentrations of molecular oxygen, the spatial profile of photon emission in the middle strip of the image was used. Under aerobic conditions, the spatial profile of photon emission shows the maximum photon emission of 17 counts. The replacement of air by nitrogen gas in the gas chamber caused a
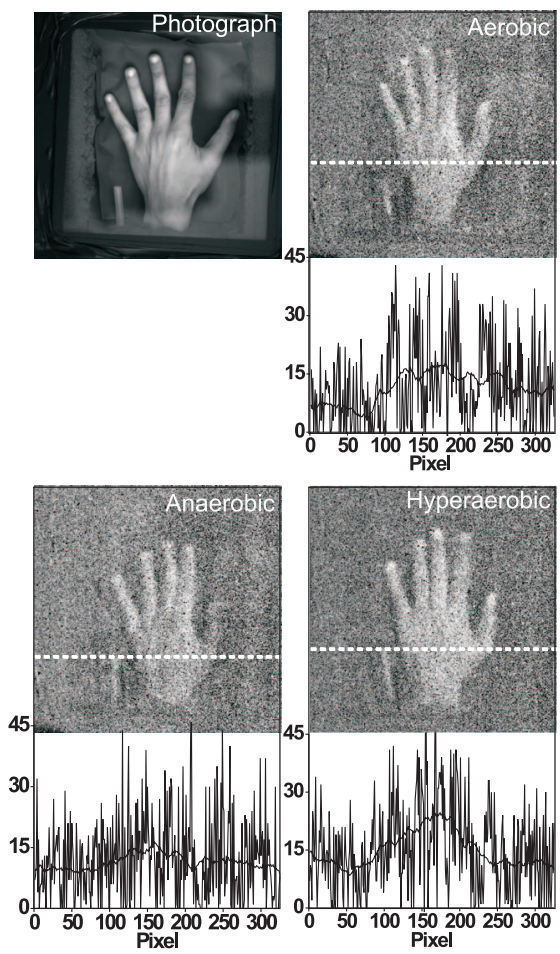

Fig. 4 Effect of molecular oxygen on two-dimensional spontaneous ultraweak photon emission from the dorsal side of the hand. The bottom panel shows the spatial profile of photon emission in the middle strip of the image. $Y$ axis denotes the number of counts accumulated after $30 \mathrm{~min}$, whereas the $X$ axis denotes the pixel of the image. The other experimental conditions were the same as in Fig. 3.

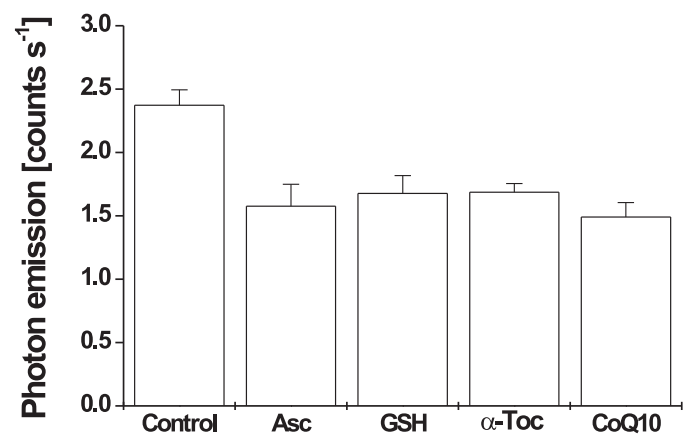

Fig. 5 Effect of ROS scavengers on one-dimensional spontaneous ultraweak photon emission from the dorsal side of the hand. Prior to the measurement the hydrophilic (ascorbate and gluthatione) and the lipophilic ( $\alpha$-tocopherol and coenzyme Q10) ROS scavengers at the concentration of $5 \mathrm{mM}$ were topically applied on the the dorsal side of the hand. Spontaneous ultraweak photon emission from the human hand was measured for $15 \mathrm{~min}$. Each bar represents the mean value of at least three independent measurements \pm SD made at the same diurnal time of three different days.

decrease in the photon emission to 12 counts, whereas the supply of molecular oxygen in the gas chamber resulted in the enhancement in photon emission to 25 counts. These observations show that molecular oxygen is involved in the two-dimensional spontaneous ultraweak photon emission, which provides valuable information on the spatial distribution of electronically excited species formed during the oxidative metabolic processes in the epidermal cells of the skin.

\subsection{Effect of Reactive Oxygen Species Scavengers on One-Dimensional Spontaneous Ultraweak Photon Emission}

To study the involvement of ROS in ultraweak photon emission, the effect of various ROS scavengers on spontaneous ultraweak photon emission from the human hand was measured. When hydrophilic (sodium ascorbate and glutathione) and lipophilic ( $\alpha$ tocopherol and coenzyme Q10) ROS scavengers were topically applied on human skin, the decrease in spontaneous ultraweak photon emission to 1.6 counts s$^{-1}$ (by $30 \%$ compared to control) was observed (Fig. 5). To confirm that the decrease in photon emission is not a pure effect of the solvent, the effect of water and ethanol on photon emission from human skin was tested. There was no measurable difference observed when only distilled water or ethanol was topically applied on the human hand (data not shown). These results indicate that ROS are involved in spontaneous ultraweak photon emission from human skin. The observation that suppression of spontaneous ultraweak photon emission with the topical application of hydrophilic or lipophilic scavengers was found to be approximately the same, indicates that the solubility of scavenger does not considerably affect the scavenging property of topically applied scavengers.

\subsection{Effect of Reactive Oxygen Species Scavengers on Two-Dimensional Spontaneous Ultraweak Photon Emission}

In further study, the effect of ROS scavengers on twodimensional spontaneous ultraweak photon emission from the 

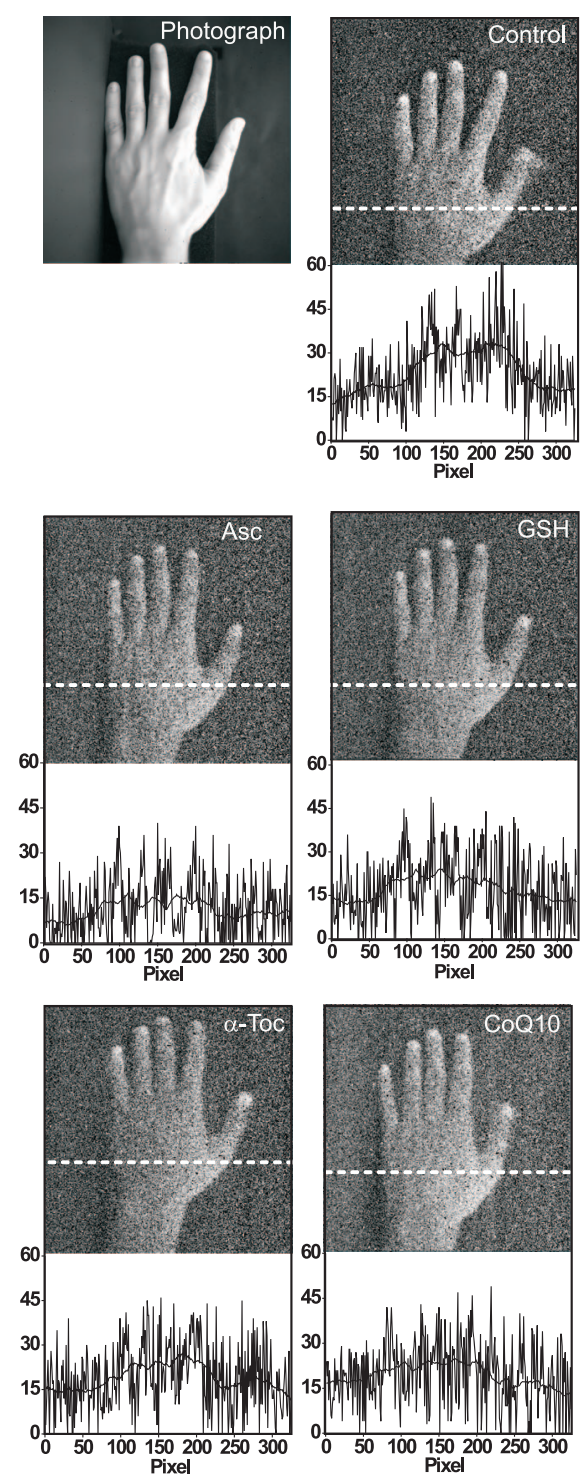

Fig. 6 Effect of ROS scavengers on two-dimensional spontaneous ultraweak photon emission from the dorsal side of the hand. The bottom panel shows the spatial profile of photon emission in the middle strip of the image. The $Y$ axis denotes the number of counts accumulated after $30 \mathrm{~min}$, whereas the $X$ axis denotes the pixel of the image. The other experimental conditions were the same as in Fig. 5.

human hand was measured using a CCD camera. Figure 6 shows the photograph of the human hand and corresponding photon emission images measured on the dorsal side of the hand without scavengers (control) and after topical application of hydrophilic (sodium ascorbate and glutathione) and lipophilic ( $\alpha$-tocopherol and coenzyme Q10) ROS scavengers. In the control sample, the spatial profile of photon emission shows the maximum photon emission of 30 counts. The spontaneous ultraweak photon emission is higher compared to the photon emission observed in the gas chamber (Fig. 4) due to the lack of photon absorption by the glass plate in the gas chamber. The topical application of hydrophilic (sodium ascorbate and glutathione) and lipophilic ( $\alpha$-tocopherol and coenzyme Q10) ROS scavengers caused a decrease in the photon emission to about 20 counts. The observation that a topical application of water and ethanol on the dorsal side of the hand has no effect on spontaneous ultraweak photon emission, indicates that the decrease in photon emission is solely caused by the scavenging (data not shown). These observations reveal that ROS are involved in the two-dimensional spontaneous ultraweak photon emission, monitoring the spatial distribution of ROS in the oxidative metabolic processes in the epidermal cells of the skin.

\section{Discussion}

In this study, the effect of molecular oxygen and various ROS scavengers on spontaneous ultraweak emission from human skin was studied using highly sensitive PMT and CCD camera. The direct evidence is provided on the involvement of molecular oxygen and ROS in the spontaneous ultraweak emission from human skin. Based on the observation that removal of molecular oxygen and topical application of $\mathrm{O}_{2} \cdot{ }^{-}$scavengers on human skin decreased ultraweak photon emission, it is concluded that $\mathrm{O}_{2}{ }^{--}$formed during oxidative metabolic processes plays a crucial role in the spontaneous ultraweak photon emission from human skin. The direct contact of human skin with atmospheric molecular oxygen and the reduction of molecular oxygen by heme-contaning enzymes in the mitochondrial membrane or cytoplasm are discussed as a possible mechanism responsible for the involvement of $\mathrm{O}_{2}{ }^{--}$in spontaneous ultraweak photon emission from human skin.

It is well accepted that skin as the outermost layer of the body is in direct contact with the atmospheric gases, such as molecular nitrogen and potentially deleterious molecular oxygen. The exchange of molecular oxygen between epidermal cell and the surrounding atmosphere proceeds via cutaneous respiration. ${ }^{37}$ At normal rest and ordinary atmospheric conditions, the rate of cutaneous respiration in a human is 1 to $1.5 \%$ of pulmonary respiration. ${ }^{38}$ As the epidermal cells in the stratum corneum absorb the molecular oxygen directly from the atmosphere, the probability of ROS formation by respiration process is higher in the stratum corneum in comparison to other layers of the skin. The data presented in this study shows that molecular oxygen is involved in spontaneous ultraweak photon emission from human skin (Figs. 3 and 4).

It is well known that epidermal cells are continuously renewed with a high turnover rate utilizing reservoirs of stem cells located in the basal layer. ${ }^{39}$ It has been observed that under in vitro conditions, the mitochondrial activity increases in the epithelial cell with high proliferation. ${ }^{40}$ It seems likely that enhancement in the mitochondrial activity increases the probability of electron leakage to molecular oxygen and production of $\mathrm{O}_{2} \cdot-$. In addition to the mitochondrial electron transport chain, the skin karatinocytes cell contains a special cytoplasmic enzyme, i.e., heme-flavoprotein NOX known to reduce molecular oxygen to $\mathrm{O}_{2} \cdot-\cdot{ }^{10}$

To prevent a deleterious effect of $\mathrm{O}_{2}{ }^{-}$formed during the normal metabolic processes in the epidermal cells, it has developed effective nonenzymatic and enzymatic antioxidant systems. ${ }^{11}$ Nonenzymatic antioxidant system in human skin includes hydrophilic (ascorbate and gluthatione) and lipophilic ( $\alpha$ tocopherol and coenzyme Q10) antioxidants. ${ }^{13}$ The observation that the topical application of both hydrophilic and lipophilic $\mathrm{O}_{2} \cdot{ }^{-}$scavengers on human skin decreased the photon emission, reveals that $\mathrm{O}_{2} \cdot-$ is involved in spontaneous ultraweak 
photon emission from human skin (Figs. 5 and 6). Ascorbate, glutathione, $\alpha$-tocopherol, and coenzyme Q10 were reported to penetrate in the skin with the following efficiency: $100 \%$ stratum corneum $>20 \%$ viable layer of epidermis $>2 \%$ dermis. ${ }^{41-43}$ It has been previously established that these are predominantly reduced antioxidants that effectively eliminate $\mathrm{O}_{2} \cdot{ }^{-}$by its reduction to hydrogen peroxide $\left(\mathrm{H}_{2} \mathrm{O}_{2}\right){ }^{44,45}$ The oxidation of hydrophilic ascorbate and gluthatione by $\mathrm{O}_{2} \cdot{ }^{-}$results in the formation of monodehydroascorbate and glutathione disulfide, respectively, whereas the oxidation of lipophilic $\alpha$ tocopherol and coenzyme Q10 by $\mathrm{O}_{2}{ }^{-}$forms $\alpha$-tocopheryl and ubisemiquinone, respectively. ${ }^{46,47}$

To maintain continuous scavenging of $\mathrm{O}_{2}{ }^{-}$by reduced scavengers, re-reduction of oxidized antioxidants has to be maintained. The re-reduction of an oxidized antioxidant proceeds either by nonenzymatic or enzymatic reactions. In the nonenzymatic reaction, self-reaction of monodehydroascorbate forms ascorbate and dehydroascorbate, whereas $\alpha$-tocopherol is recovered by the reduction of $\alpha$-tocopheryl by ascorbate. ${ }^{46,48}$ It has been previously reported that the epidermal cells contain a higher level of ascorbate and $\alpha$-tocopherol compared to the dermal cells of the skin. ${ }^{11,49}$ In the enzymatic reaction, the rereduction of oxidized antioxidants is catalyzed by various types of NADPH-reductase. ${ }^{2}$ The reduction of glutathione disulfide to glutathione is catalyzed by NADPH-glutathione reductase, ${ }^{50}$ whereas NADPH quinine reductase converts ubisemiquinone to ubiquinol. ${ }^{41}$ It has been previously reported that the activity of NADPH-glutathione reductase ${ }^{11}$ and NADPH quinine reductase $\mathrm{e}^{47}$ in the epidermis cells is several times higher compared to the dermis cells. Enzymatic scavenging is maintained by the peroxidase family of enzymes such as glutathione peroxidases and catalase. ${ }^{49}$ Glutathione peroxidase catalyzes decomposition of $\mathrm{H}_{2} \mathrm{O}_{2}$ in the water and molecular oxygen using glutathione as a substrate, whereas catalase catalyzes the decomposition of $\mathrm{H}_{2} \mathrm{O}_{2}$ in the water and molecular oxygen. It has been previously shown that catalase activity is several orders higher in the epidermal cells when compared to the dermal cells of the skin. ${ }^{11,51}$

In this study, the ultraweak photon emission is demonstrated as a nondestructive technique for the monitoring of oxidative metabolic processes in the epidermal cells of the skin. It is proposed here that ultraweak photon emission can serve as a reliable and relevant method for the noninvasive determination of antioxidant capacity in human skin. The application of ultraweak photon emission in the clinical studies opens the area for the use of ultraweak photon emission as a highly sensitive and fast diagnostic tool for the early detection of various chronic skin diseases and skin aging processes in the medicine and the cosmetic industry.

\section{Acknowledgments}

This work was supported by the Centre of the region Haná for biotechnological and agricultural research (Grant No. CZ.1.05/2.1.00/01.0007), the Ministry of Education, Youth and Sports of Czech Republic (Grant No. MSM6198959215), and the student project PrF_2010_050 of the Palacký University. We thank to Ankush Prasad for his help with data collection and Dr. Pavel Krchňák for technical assistance with respect to PMT and CCD camera measurements.

\section{References}

1. V. J. Thannickal and B. L. Fanburg, "Reactive oxygen species in cell signaling," Am. J. Physiol. Lung Cell Mol. Physiol. 279, L1005-L1028 (2000), http://www.ncbi.nlm.nih.gov/pubmed/11076791.

2. B. Halliwell and J. M. C. Gutteridge, in Free Radicals in Biology and Medicine, 4th ed., Oxford University, London (2007).

3. J. P. Spencer, A. Jenner, K. Chimel, O. I. Aruoma, C. E. Cross, R. Wu, and B. Halliwell, "DNA strand breakage and base modification induced by hydrogen peroxide treatment of human respiratory tract epithelial cells," FEBS Lett. 374, 233-236 (1995).

4. J. Krutmann, "Ultraviolet A radiation-induced biological effects in human skin: relevance for photoaging and photodermatosis," J. Dermatol. Sci. 23, S22-S26 (2000).

5. M. Kunisada, H. Kumimoto, K. Ishizaki, K. Sakumi, Y. Nakabeppu, and C. Nishigori, "Narrow-band UVB induces more carcinogenic skin tumors than broad-band uvb through the formation of cyclobutane pyrimidine dimer," J. Invest. Dermatol. 127, 2865-2871 (2007).

6. G. Lenaz, "Role of mitochondria in oxidative stress and ageing," Biochim. Biophys. Acta 1366, 53-67 (1998).

7. S. Raha and B. H. Robinson, "Mitochondria, oxygen free radicals, disease and ageing," TIBS 25, 502-508 (2000).

8. G. R. Aitken, J. R. Henderson, S.-C. Chang, C. J. McNeil, and M. A. Birch-Machin, "Direct monitoring of UV-induced free radical generation in HaCaT keratinocytes," Clin. Exp. Dermatol. 32, 722-727 (2007).

9. M. A. Birch-Machin and H. Swalwell, "How mitochondria record the effects of UV exposure and oxidative stress using human skin as a model tissue," Mutagenesis 25, 101-107 (2010).

10. W. Chamulitrat, W. Stremmel, T. Kawahara, K. Rokutan, H. Fujii, K. Wingler, H. H. H. W. Schmidt, and R. Schmidt, "A constitutive NADPH oxidase-like system containing gp91phox homologs in human keratinocytes," J. Invest. Dermatol. 122, 1000-1009 (2004).

11. Y. Shindo, E. Witt, D. Han, W. Epstein, and L. Packer, "Enzymic and non-enzymic antioxidents in epidermis and dermis of human skin," $J$. Invest. Dermatol. 102, 122-124 (1994).

12. B. Allemann and L. Baumann, "Antioxidents used in skin care formulations," Skin Therapy Letter 13, 5-9 (2008), http://www.ncbi. nlm.nih.gov/pubmed/18839043.

13. H. Masaki, "Role of antioxidents in skin: anti-ageing effects," J. Dermatol. Sci. 58, 85-90 (2010).

14. S. Briganti and M. Picardo, "Antioxidant activity, lipid peroxidation and skin diseases. What's new," J. Eur. Acad. Dermatol. Venereol. 17, 663-669 (2003).

15. N. Duran and E. Cadenas, "The role of singlet oxygen and triplet carbonyls in biological systems," Rev. Chem. Intermed. 8, 147-187 (1987).

16. G. Cilento and I. L. Brunetti, "Triplet carbonyls: from photophysics to biochemistry," J. Mol. Struct. 324, 45-48 (1994).

17. M. Kobayashi, M. Usa, and H. Inaba, "Highly sensitive detection and spectral analysis of ultraweak photon emission from living samples of humans origin for the measurement of biomedical information," Trans. Soc. Instrument. Control Eng. E-1, 214-220 (2001) http://srv01.sice.or.jp/ e-trans/papers/E1-27.pdf.

18. C. Kageyama, K. Kato, H. Iyozumi, H. Inagaki, A. Yamaguchi, K. Furuse, and K. Baba, "Photon emissions from rice cells elicited by $\mathrm{N}$-acetylchitooligosaccharide are generated through phospholipid signaling in close association with the production of reactive oxygen species," Plant. Physiol. Biochem. 44, 901-909 (2006).

19. B. S. Cheun, S. H. Yi, K. Y. Baik, J. K. Lim, J. S. Yoo, H. W. Shin, and K. S. Soh, "Biophoton emission of MDCK cell with hydrogen peroxide and $60 \mathrm{~Hz}$ AC magnetic field," J. Environ. Bio. 28, 735-740 (2007), http://www.ncbi.nlm.nih.gov/pubmed?term $=18405105 \% 20$.

20. R. V. Wijk, E. P. A. V. Wijk, F. A. C. Wiegant, and J. Evis, "Free radicals and low-level photon emission in human pathogenesis: state of the art," Indian J. Exp. Biol. 46, 273-309 (2008), http://www.ncbi. nlm.nih.gov/pubmed?term $=18697612 \% 5$ Buid\%5D.

21. A. Rastogi and P. Pospísil, "Effect of exogenous hydrogen peroxide on biophoton emission from radish root cells," Plant. Physiol. Biochem. 48, 117-123 (2010).

22. K. Lieboda, D. Fabler, W. D. Schmidt, T. Kühn, and U. Wollina, "In vivo spectroscopy in dermatology: methods and new fields of application," J. Eur. Acad. Dermatol. Venereol. 14, 1-4 (2000). 
23. M. Takeda, M. Kobayashi, M. Takayama, S. Suzuki, T. Ishida, K. Ohnuki, T. Moriya, and N. Ohuchi, "Biophoton detection as a novel technique for cancer imaging," Cancer Sci. 95, 656-661 (2004).

24. L. Brancaleon, M. P. Bamberg, T. Sakamki, and N. Kollias, "Attenuated total reflection-Fourier transform infrared spectroscopy as a possible method to investigate biophysical parameters of stratum corneum in vivo," J. Invest. Dermatol. 116, 380-386 (2001).

25. M. E. Darvin, W. Sterry, and J. Lademann, "Resonance Raman spectroscopy as an effective tool for the determination of antioxidative stability of cosmetic formulation," J. Biophoton. 3, 82-88 (2010).

26. J. Lademann, S. Schanzer, M. Meinke, W. Sterry, and M. E. Darvin, "Interaction between carotenoids and free radicals in human skin," Skin Pharmacol. Physiol. 24, 238-244 (2011).

27. J. W. Fluhr, P. Caspers, J. A. V. D. Pol, H. Richter, W. Sterry, J. Lademann, and M. E. Darvin, "Kinetics of carotenoid distribution in human skin in vivo after exogenous stress: disinfectant and wIRA-induced carotenoid depletion recovers from outside to inside," J. Biomed. Opt. 16, 035002-1-035002-7 (2011).

28. M. E. Darvin, J. W. Fluhr, P. Caspers, A. V. D. Pool, H. Richter, A. Patzelt, W. Sterry, and J. Lademann, "In vivo distribution of carotenoids in different anatomical locations of human skin: comparative assessment with two different Raman spectroscopy methods" Exp. Dermatol. 18, 1060-1063 (2009).

29. A. Jain, I. Rieger, M. Rohr, and A. Schrader, "Antioxidant efficieny on human skin in vivo investigated by UVA-induced chemiluminescence decay analysis via induced chemiluminescence human skin," Skin Pharmacol. Physiol. 23, 266-272 (2010).

30. A. Rastogi and P. Pospíśil, "Ultra-weak photon emission as a noninvasive tool for monitoring of oxidative processes in the epidermal cells of human skin: comparative study on the dorsal and the palm side of the hand," Skin Res. Technol. 16, 365-370 (2010).

31. R. Hagens, F. Khabiri, V. Schreiner, H. Wenck, K.-P. Wittern, H.-J. Duchstein, and W. Mei, "Non-invasive monitoring of oxidative skin stress by ultraweak photon emission measurement. II: biological validation on ultraviolet stressed skin," Skin Res. Technol. 14, 112-120 (2008).

32. H. Kim, S. Ahn, and J. Kim, "Biophoton emission induced by ultrasonic irradiation," IFMBE Proceedings 14(8), 1277-1280 (2007).

33. H. Ou-Yang, G. Stamatas, C. Saliouand, and N. Kollias, "A Chemiluminescence study of UVA-induced oxidative stress in human skin in vivo" J. Invest. Dermatol. 122, 1020-1029 (2004).

34. R. V. Wijk, M. Kobayashi, and E. P. A. V. Wijk, "Anatomic characterization of human ultra-weak photon emission with a moveable photomultiplier and CCD imaging," J. Photochem. Photobiol. B 83, 69-76 (2006).

35. M. Kobayashi, D. Kikuchi, and H. Okamura, "Imaging of ultraweak spontaneous photon emission from human body displaying diurnal rhythm," PLOS ONE 4(7), e6256 (2009).

36. H. H. Jung, J. M. Yang, W. M. Woo, C. Choi, J. S. Yang, and K. S. Soh, "Years-long biophoton measurements: normalized frequency count analysis and seasonal dependency," J. Photochem. Photobiol., B 78, 149-154 (2005).

37. C. B. Archer, "Functions of the skin," in Rook's Textbook of Dermatology, T. Burns, S. Breathnach, N. Cox, and C. Griffiths, Eds., pp. 4.1-4.11, Wiley-Blackwell, John Wiley \& Sons Ltd, Chichester, UK (2010).

38. H. Takiwaki, "Measurement of transcutaneous oxygen tension," in Handbook of Non-Invasive Methods and the Skin, J. Serup and G. B. E. Jamec, Eds., pp. 185-195, Chemical Rubber, Boca Raton (1995).

39. E. Reefman, P. C. Limburg, C. G. M. Kallenberg, and M. Bijl, "Apoptosis in human skin: role in pathogenesis of various diseases and relevance for therapy," Ann. N.Y. Acad. Sci. 1051, 52-63 (2005).

40. K. Takeda, S. Akagi, S. Takahashi, A. Onishi, H. Hanada, and C. A. Pinkert, "Mitochondrial activity in response to serum strvation in bovaine (Bos taurus) cell culture," Cloning and Stem Cells 4, 223-229 (2002), http://www.ncbi.nlm.nih.gov/pubmed/12398803.

41. U. Hoppe, J. Bergemann, W. Diembeck, J. Ennen, S. Gohla, I. Harris, J. Jocob, J. Kielholz, W. Mei, D. Pollet, D. Schachtschabel, G. Sauermann, V. Schreiner, F. Stab, and F. Steckel, "Coenzyme Q10, a cutaneous antioxidant and energizer," BioFactors 9, 371-378 (1999).

42. R. M. W. Moison and G. M. J. B. V. Henegouwen, "Topical antioxidant vitamins $\mathrm{C}$ and $\mathrm{E}$ prevent UVB-radiation-induced peroxidation of eicosapentaenoic acid in pig skin," Radiat. Res. 157, $402-409$ (2002).

43. J. M. Rijnkels, R. M. W. Moison, E. Podda, and G. M. J. B. V. Henegouwen, "Photoprotection by antioxidants against UVB-radiationinduced damage in pig skin organ culture," Radiat. Res. 159, 210-217 (2003).

44. A. Nandi and I. B. Chatterjee, "Scavenging of superoxide radical by ascorbic acid," J. Biosci. 11, 435-441 (1987).

45. E. Damiani, P. Astolfi, P. Carloni, P. Stipa, and L. Greci, "Antioxidents: how they work," in Oxidants in Biology, G. Valacchi and P. A. Davis, Eds. pp. 251-266, Springer Science, New York (2008).

46. L. Packer, S. U. Weber, and G. Rimbach, "Molecular aspect of $\alpha$-tocoptrienol antioxidant action and cell signaling," J. Nutr. 131, 369S-373S (2001), http://www.ncbi.nlm.nih.gov/pubmed?term= $11160563 \% 5$ Buid\%5D.

47. M. Takada, T. Yuzuriha, and C. Yamato, "Redox levels of intravenously administered $\left[{ }^{14} \mathrm{C}\right]$ Coenzyme Q10 and Coenzyme Q10reducing activity in subcellular fractions of guinea pig liver," $J$. Nutr. Sci. Vitaminol. (Tokyo) 31, 147-155 (1985), http://www.ncbi. nlm.nih.gov/pubmed?term $=3928843 \% 5$ Buid\%5D.

48. G. Banhegyi, L. Braun, M. Csala, P. Ferenc, and M. Jozsef, "Ascorbate metabolism and its regulation in animals," Free Radic. Biol. Med. 23, 793-803 (1997)

49. M. Lopez-Torres, J. J. Thiele, Y. Shindo, D. Han, and L. Packer, "Topical application of $\alpha$-tocopherol modulates the antioxidant network and diminishes ultraviolet-induced oxidative damage in murine skin," $B r . J$. Dermatol. 138, 207-215 (1998).

50. O. Ortolani, A. Conti, A. R. De Gaudio, E. Moraldi, Q. Cantini, and G. Novelli, "The effect of glutathione and N-acetylcysteine on lipoperoxidative damage in patients with early septic shock," $\mathrm{Am}$. J. Respir. Crit. Care. Med. 161, 1907-1911 (2000) http://www.ncbi. nlm.nih.gov/pubmed?term $=10852765$.

51. G. E. Rhie, M. H. Shin, J. Y. Seo, W. W. Choi, K. H. Cho, K. H. Kim, K. C. Park, H. C. Eun, and J. H. Chung, "Aging- and photoageingdependent changes of enzymic and nonenzymic antioxidents in the epidermis and dermis of human skin in vivo," J. Invest. Dermatol. 117, $1212-1217$ (2001) 